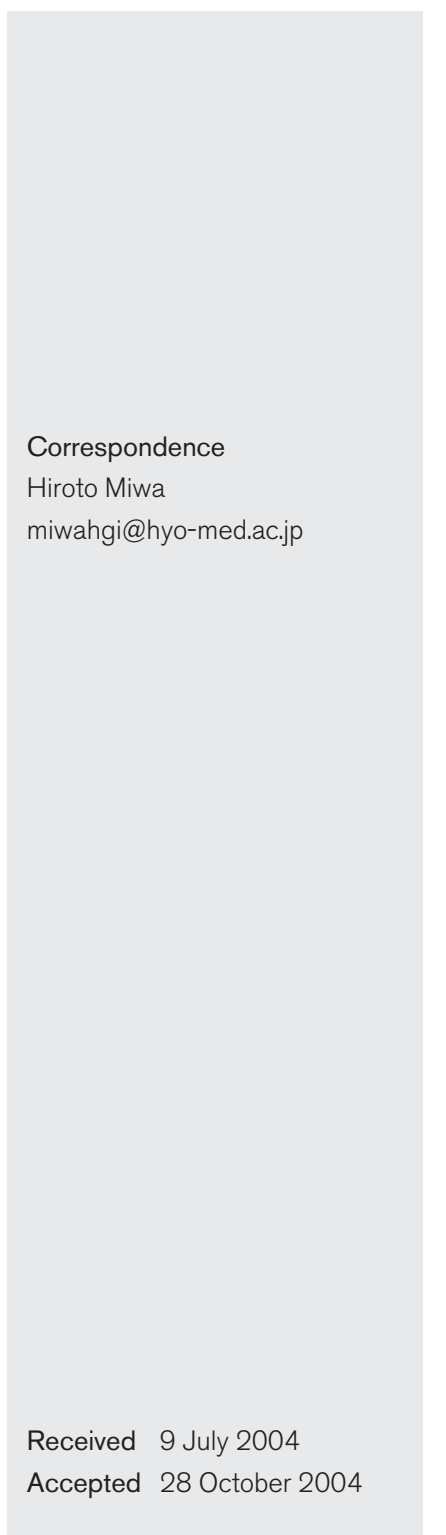

\title{
Influence of antimicrobial treatment for Helicobacter pylori infection on the intestinal microflora in Japanese macaques
}

\author{
Junji Tanaka, ${ }^{1}$ Yoshihiro Fukuda, ${ }^{1}$ Shigeyuki Shintani, ${ }^{1}$ Kazutoshi Hori, ${ }^{1}$ \\ Toshihiko Tomita, ${ }^{1}$ Toshifumi Ohkusa, ${ }^{2}$ Takayuki Matsumoto ${ }^{1}$ \\ and Hiroto Miwa ${ }^{1}$ \\ ${ }^{1}$ Division of Gastroenterology, Department of Internal Medicine, Hyogo Medical College, \\ Mukogawa-cho 1-1, Nishinomiya, Hyogo 663-8501, Japan \\ ${ }^{2}$ Division of Gastroenterology, Department of Internal Medicine, Juntendo University, School of \\ Medicine, Tokyo, Japan
}

\begin{abstract}
Eradication treatment for Helicobacter pylori is known to cause mild but relatively frequent adverse effects. Some adverse effects such as diarrhoea and soft stools are related to disruption of the composition of the intestinal microflora. This study investigated the microfloral changes resulting from administration of an eradication regimen using proton pump inhibitor (PPI), amoxycillin and clarithromycin. Twenty-eight laboratory-bred Japanese macaques either were administered eradication treatment by this regimen for 7 days or received no medication. Faecal samples were collected for analysis on days 0,8 and 15 , and both aerobic and anaerobic cultures were performed. Among aerobic bacteria, Streptococcus had significantly decreased by day 8 , while Enterococcus and Enterobacteriaceae had significantly increased. However, the total number of aerobic bacteria was not significantly decreased from pretreatment levels 1 day after completion of treatment. The number of anaerobic bacteria did not change significantly by day 8 . However, the number of Lactobacillus and the detection rate of Bifidobacterium, Peptostreptococcus and Veillonella significantly decreased by day 8 , although the number of Bifidobacterium, Peptostreptococcus and Veillonella had almost recovered up to the pretreatment levels 1 week after completion of treatment (day 15). These results suggest that the alterations in the composition of the intestinal microflora caused by the antimicrobial regimen that excludes metronidazole are different from those caused by the regimen including this drug. However, the alterations in bacterial microflora had almost reversed 7 days after completion of treatment in these macaques, which supports clinical findings that diarrhoea or soft stools in humans resolve relatively quickly after a similar treatment.
\end{abstract}

\section{INTRODUCTION}

Advancements in the understanding of the pathogenicity of Helicobacter pylori infections in various gastric diseases, including peptic ulcers (Van der Hulst et al., 1997), mucosa-associated lymphoid tissue lymphoma (Wotherspoon et al., 1993) and gastric cancer (Uemura et al., 2001), have increased the opportunities for utilizing eradication treatment for this organism. Proton pump inhibitor (PPI)-based triple therapies are the most popular and effective eradication regimens and are used worldwide (Sharma et al., 1999). This combination of drugs is known to cause mild but relatively frequent adverse effects (Lind et al., 1996; Misiewicz et al., 1997). Such side effects are mostly attributed to the use of antimicrobials, and some of the side effects such

Abbreviation: PPI, proton pump inhibitor. as diarrhoea and soft stools are related to alterations in intestinal microflora (Armuzzi et al., 2001; Bartlett, 2002; Cremonini et al., 2002; Högenauer et al., 1998; Sullivan et al., 2001).

Analysis of profiles of the intestinal microflora would be useful in exploring the mechanisms of the diarrhoea associated with this treatment. A few reports have described microfloral changes during and after eradication treatment by regimens containing PPI and metronidazole (Adamsson et al., 1999; Bühling et al., 2001). However, publications focusing on microfloral changes brought about by the combination regimen of PPI, amoxycillin and clarithromycin treatment, which is the only regimen approved by the Japanese social security foundation (Asaka et al., 2001), are not available. 
On the other hand, it is not easy to investigate changes in intestinal microflora after eradication treatment in humans due to methodological limitations such as difficult sampling procedures and the influence of food or environmental factors on intestinal microflora. Such methodological difficulties could be overcome using experimental animal models. Therefore, in this study, we investigated changes in intestinal microflora resulting from administration of an eradication regimen using PPI, amoxycillin and clarithromycin in Japanese macaques.

\section{METHODS}

Animals. Twenty-eight laboratory-bred Japanese macaques, aged 5-6 years (body weight $8 \cdot 2-9 \cdot 7 \mathrm{~kg}$ ), were used. The animals were kept in a room exclusively used for macaques with the temperature set at 20 $25^{\circ} \mathrm{C}$, humidity adjusted to $50-75 \%$, ventilation not less than 10 times per hour and illumination by artificial lighting for $8 \mathrm{~h}$ per day. Animal cages and rooms were cleaned daily, and the floor was disinfected using TEGO-51 (Nihon Shoji) (Fukuda et al., 1992). All the macaques that were not infected with $H$. pylori were maintained in separate, isolated cages to prevent contamination via saliva and excreta. The animals were fed commercially available PS solid (Oriental Yeast Industries) and were given free access to tap water from a water bottle (Fukuda et al., 1992).

Drug administration and stool sampling. Twenty animals were given the eradication treatment by the PPI/amoxycillin/clarithromycin regimen for 7 days, and eight macaques received no treatment (control animals). Briefly, after animals had fasted more than $12 \mathrm{~h}$ (no water restriction), a capsule containing $30 \mathrm{mg}$ of lansoprazole, six capsules containing $1500 \mathrm{mg}$ of amoxycillin and two tablets containing of $400 \mathrm{mg}$ clarithromycin were orally administered to anaesthetized macaques once daily for 7 days (treatment group). Preparation for anaesthetization was done with intramuscular ketamine hydrochloride designed for animal use (Sankyo) at a dose of $10 \mathrm{mg} \mathrm{kg}^{-1}$ (Fukuda et al., 1992). After dosing, the anaesthetized macaques were endoscoped by a sterilized fibrescope GIF- $\mathrm{P}_{10}$ (Olympus Optical) to confirm that these drugs were in the stomach (Fukuda et al., 1992).
In both the treatment and control groups, stool samples were collected before (day 0), 1 day after (day 8) and 1 week after the dosing (day 15). Faeces of each macaque were collected directly from the rectum by a sterilized stick of our making.

Analysis of the intestinal microflora profiles. Immediately after being obtained, stools were packed in a sterile vinyl bag and mixed well to attain a homogeneous state. Part of the stool (about $2 \mathrm{~g}$ ) was taken from the centre portion of the mixed faeces, placed in a transport tube (SEEDTUBE Eiken, Eiken Chemical Co.) and kept at $4{ }^{\circ} \mathrm{C}$.

The stool samples were transported to the hospital laboratory and were immediately processed for stool culture according to the method of Mitsuoka (1980). Briefly, an accurately weighed $1 \mathrm{~g}$ portion of faecal specimen was mixed well and diluted 10 -fold in $9 \mathrm{ml}$ of an anaerobic diluent. The composition of a $990 \mathrm{ml}$ quantity of the anaerobic diluent was as follows: $37.5 \mathrm{ml}$ salt diluent $1\left(0.78 \% \mathrm{~K}_{2} \mathrm{HPO}_{3}\right), 37.5 \mathrm{ml}$ salt diluent $2\left(0 \cdot 47 \% \mathrm{KH}_{2} \mathrm{PO}_{4}, 1 \cdot 18 \% \mathrm{NaCl}, 1 \cdot 20 \%\left(\mathrm{NH}_{4}\right)_{2} \mathrm{SO}_{4}, 0 \cdot 12 \%\right.$ $\mathrm{CaCl}_{2}, 0 \cdot 25 \% \mathrm{MgSO}_{4} \cdot \mathrm{H}_{2} \mathrm{O}$ ), $1 \mathrm{ml} 0 \cdot 1 \%$ Resazurin diluent, $0.5 \mathrm{~g}$ L-cysteine. $\mathrm{HCl}-\mathrm{H}_{2} \mathrm{O}, 2 \mathrm{ml} 25 \% \mathrm{~L}$-ascorbic acid diluent, $50 \mathrm{ml} 8 \%$ $\mathrm{Na}_{2} \mathrm{CO}_{3}$ diluent, $0.5 \mathrm{~g}$ agar and $860 \mathrm{ml}$ water. The suspension was diluted serially by 10 -fold with the same diluent up to $10^{8}$ dilution.

Aliquots $(0.05 \mathrm{ml})$ of each diluted sample were inoculated on 15 different appropriate agar media, which are shown in Table 1, and incubated under the respective conditions required for optimal growth of each organism. All aerobic plates were incubated at $35^{\circ} \mathrm{C}$ for $2-3$ days and all anaerobic plates were incubated in an anaerobic chamber (Mitsubishi Gas Chemical Co.) at $35^{\circ} \mathrm{C}$ for $3-5$ days. After incubation, different colony types were counted and identified to the genus level on the basis of Gram staining, general morphology, biochemical tests and a growth test under aerobic conditions utilizing methods described elsewhere (Murray et al., 1999). The concentration was defined as the number of colony forming units (c.f.u.) per gram (g) stool sample. The detection limit was $10^{2}$ c.f.u. (g stool $)^{-1}$.

Physical status of the macaques during the experiment. The general condition (facial expression, appearance and behaviour) and stool condition (diarrhoea or soft stool) of the animals were observed and recorded throughout the experimental period. Body weights were measured weekly under anaesthesia.

Table 1. Culture media used for isolation of intestinal bacteria

\begin{tabular}{|lll|}
\hline Medium & Organism(s) cultured & \\
\hline $\begin{array}{l}\text { Aerobic cultures } \\
\text { Sheep Blood agar M58 }\end{array}$ & Non-selective aerobic & Source \\
CLED medium & Non-selective aerobic & Eiken Chemical Co. \\
Mannitol salt agar & Staphylococcus & Nissui Pharmaceutical Co. \\
EF agar & Enterococcus & Nissui Pharmaceutical Co. \\
DHL agar & Enterobacteriaceae & Nissui Pharmaceutical Co. \\
CHROMagar Candida & Yeast & Nippon Becton Dickinson \\
NAC agar & Pseudomonas aeruginosa & Eiken Chemical Co. \\
Anaerobic cultures & & \\
BL agar & Non-selective anaerobic & Eiken Chemical Co. \\
Brucella HK agar & Non-selective anaerobic & Kyokuto Pharmaceutical Industrial Co. \\
BBE agar & Bacteroides fragilis & Kyokuto Pharmaceutical Industrial Co. \\
Kanamycin CW agar & Clostridium & Nissui Pharmaceutical Co. \\
CCFA agar & Clostridium difficile & Oxoid \\
LBS modified agar & Lactobacillus & Nippon Becton Dickinson \\
FM modified agar & Fusobacterium & Nissui Pharmaceutical Co. \\
BD Difco Veillonella agar & Veillonella & BD Diagnostic Systems \\
\hline
\end{tabular}


Statistics. Student's $t$-test, Fisher's exact test and one-way analysis of variance were used to determine the statistical significance of the number of each bacterium and the detection rates between day 0 , day 8 and day 15. Statistical significance was established at the $P<0 \cdot 05$ level.

Ethics. This study was performed in accordance with the international guiding principles for biomedical research involving animals for the prevention of cruelty to animals and was approved by the ethical committee at our medical college.

\section{RESULTS}

\section{Analysis of the intestinal microflora profiles}

The number of intestinal micro-organisms and their detection rate before and 7 and 14 days after completion of the treatment in the treated animals are shown in Table 2 , and the profile of the intestinal micro-organisms in the control macaques are depicted in Table 3 . In the control macaques, no statistically significant difference was noted in the number of intestinal micro-organisms at day 0 and day 8 , assuring the reliability of our analysis.

Regarding aerobic bacteria, Streptococcus significantly decreased just after treatment, while Enterococcus and Enterobacteriaceae significantly increased. However, the total number of aerobic bacteria was not significantly decreased on day 8 . On day 15 , the number of aerobic bacteria had increased, which might be due to an increase in bacteria that were resistant to the antimicrobials used in this study.

Regarding anaerobic bacteria, the number of Lactobacillus and the detection rate of Bifidobacterium, Peptostreptococcus and Veillonella significantly decreased during eradication treatment. In contrast, the number of Bacteroidaceae did not change, with the result that the total number of anaerobic bacteria was not statistically significantly changed, as Bacteroidaceae were in the majority among bacterial flora. Lactobacillus, Bifidobacterium, Peptostreptococcus and Veillonella

Table 2. Intestinal microflora profiles before and after eradication therapy with lansoprazole, amoxycillin and clarithromycin administration in macaques

\begin{tabular}{|c|c|c|c|c|c|c|}
\hline Organism & $\begin{array}{c}\text { No. of organisms } \\
\text { on day } 0^{*}\end{array}$ & $\begin{array}{c}\text { No. of organisms } \\
\text { on day } 8^{\star}\end{array}$ & $P$ value & $\begin{array}{c}\text { No. of organisms } \\
\text { on day } 15^{\star}\end{array}$ & $P$ value & $P$ value (ANOVA) $\dagger$ \\
\hline Enterobacteriaceae & $\begin{array}{c}7 \cdot 53 \pm 1 \cdot 05 \\
\quad(95 \%)\end{array}$ & $\begin{array}{c}8 \cdot 79 \pm 0 \cdot 79 \\
(100 \%)\end{array}$ & $<0.0001$ & $\begin{array}{c}7 \cdot 98 \pm 0 \cdot 49 \\
(100 \%)\end{array}$ & $0 \cdot 0387$ & $<0.0001$ \\
\hline Enterococcus spp. & $\begin{array}{c}6 \cdot 27 \pm 1 \cdot 07 \\
(100 \%)\end{array}$ & $\begin{array}{c}7 \cdot 50 \pm 0 \cdot 75 \\
(100 \%)\end{array}$ & $<0 \cdot 0001$ & $\begin{array}{c}7 \cdot 00 \pm 0 \cdot 83 \\
(100 \%)\end{array}$ & $0 \cdot 009$ & $0 \cdot 0002$ \\
\hline Streptococcus spp. & $\begin{array}{l}8 \cdot 95 \pm 0 \cdot 70 \\
\quad(100 \%)\end{array}$ & $\begin{array}{c}7 \cdot 26 \pm 0 \cdot 85 \\
(100 \%)\end{array}$ & $<0 \cdot 0001$ & $\begin{array}{l}9 \cdot 27 \pm 1 \cdot 01 \\
\quad(100 \%)\end{array}$ & $0 \cdot 210$ & $<0.0001$ \\
\hline Stapylococcus spp. & $\begin{array}{c}5 \cdot 29 \pm 0 \cdot 60 \\
(100 \%)\end{array}$ & $\begin{array}{c}5 \cdot 19 \pm 0 \cdot 84 \\
(100 \%)\end{array}$ & $0 \cdot 646$ & $\begin{array}{c}6 \cdot 27 \pm 1 \cdot 10 \\
(100 \%)\end{array}$ & $0 \cdot 101$ & $0 \cdot 0915$ \\
\hline Total aerobes & $9 \cdot 01 \pm 0 \cdot 70$ & $8.93 \pm 0.64$ & 0.696 & $9 \cdot 42 \pm 0 \cdot 67$ & $0 \cdot 0358$ & $0 \cdot 0305$ \\
\hline Lactobacillus spp. & $\begin{array}{c}7 \cdot 78 \pm 1 \cdot 59 \\
(100 \%)\end{array}$ & $\begin{array}{c}4 \cdot 31 \pm 1 \cdot 53 \\
\quad(55 \%)\end{array}$ & $<0 \cdot 0001$ & $\begin{array}{c}7 \cdot 11 \pm 1 \cdot 87 \\
(100 \%)\end{array}$ & $0 \cdot 701$ & $<0 \cdot 0001$ \\
\hline Bifidobacterium spp. & $\begin{array}{c}8 \cdot 80 \pm 0 \cdot 76 \\
\quad(40 \%)\end{array}$ & $\begin{array}{l}<0 \cdot 23 \\
(0 \%) \ddagger\end{array}$ & - & $\begin{array}{c}8 \cdot 44 \pm 0 \cdot 74 \\
(25 \%)\end{array}$ & - & - \\
\hline Eubacterium spp. & $\begin{array}{c}8 \cdot 88 \pm 0 \cdot 55 \\
\quad(100 \%)\end{array}$ & $\begin{array}{c}8 \cdot 69 \pm 0 \cdot 40 \\
\quad(95 \%)\end{array}$ & $0 \cdot 255$ & $\begin{array}{c}9 \cdot 19 \pm 0 \cdot 43 \\
(100 \%)\end{array}$ & $0 \cdot 019$ & $0 \cdot 0031$ \\
\hline Clostridium spp. & $\begin{array}{l}6 \cdot 96 \pm 0 \cdot 73 \\
\quad(90 \%)\end{array}$ & $\begin{array}{c}7 \cdot 24 \pm 0 \cdot 72 \\
\quad(70 \%)\end{array}$ & $0 \cdot 167$ & $\begin{array}{c}6 \cdot 89 \pm 0 \cdot 82 \\
\quad(85 \%)\end{array}$ & $0 \cdot 274$ & $0 \cdot 340$ \\
\hline Peptostreptococcus spp. & $\begin{array}{c}8 \cdot 51 \pm 0 \cdot 66 \\
\quad(80 \%)\end{array}$ & $\begin{array}{c}8 \cdot 45 \pm 0 \cdot 21 \\
\quad(10 \%) \ddagger\end{array}$ & $0 \cdot 302$ & $\begin{array}{c}8 \cdot 36 \pm 0 \cdot 61 \\
\quad(60 \%)\end{array}$ & $0 \cdot 719$ & $0 \cdot 362$ \\
\hline Veillonella spp. & $\begin{array}{c}5 \cdot 86 \pm 1 \cdot 67 \\
\quad(90 \%)\end{array}$ & $\begin{array}{l}3 \cdot 10 \\
(5 \%) \S\end{array}$ & - & $\begin{array}{c}6 \cdot 26 \pm 0 \cdot 86 \\
(90 \%)\end{array}$ & $0 \cdot 307$ & - \\
\hline Fusobacterium spp. & $\begin{array}{c}7 \cdot 17 \pm 1 \cdot 04 \\
\quad(75 \%)\end{array}$ & $\begin{array}{c}7 \cdot 28 \pm 0 \cdot 71 \\
\quad(70 \%)\end{array}$ & $0 \cdot 369$ & $\begin{array}{l}6 \cdot 85 \pm 0 \cdot 85 \\
\quad(70 \%)\end{array}$ & $0 \cdot 900$ & $0 \cdot 532$ \\
\hline Bacteroidaceae & $\begin{array}{c}9 \cdot 79 \pm 0 \cdot 56 \\
\quad(100 \%)\end{array}$ & $\begin{array}{l}9 \cdot 75 \pm 0 \cdot 45 \\
\quad(100 \%)\end{array}$ & $0 \cdot 812$ & $\begin{array}{l}9 \cdot 93 \pm 0 \cdot 60 \\
(100 \%)\end{array}$ & $0 \cdot 326$ & $0 \cdot 432$ \\
\hline Total anaerobes & $9 \cdot 97 \pm 0 \cdot 49$ & $9 \cdot 81 \pm 0 \cdot 42$ & $0 \cdot 240$ & $10 \cdot 1 \pm 0 \cdot 50$ & $0 \cdot 424$ & $0 \cdot 145$ \\
\hline
\end{tabular}

${ }^{\star}$ Number of organisms measured as (mean $\log _{10} \pm \mathrm{SD}$ ) per gram faeces. Detection incidence in all of macaques is shown in parentheses.

$\dagger P$ values for comparison among day 0 , day 8 and day 15 analysed by analysis of variances with repeated measures and post hocs.

$¥$ The detection incidence was significantly different $(P<0 \cdot 01)$.

$\S P<0.001$ from before administration analysed by Fisher’s exact test. 
Table 3. Intestinal microflora profiles in control macaques

\begin{tabular}{|lccc|}
\hline Organism & \multicolumn{2}{c}{ No. of organisms* } & \multirow{2}{*}{ P value } \\
\cline { 2 - 3 } & Day 0 & \\
\hline Aerobes & & \\
Enterobacteriaceae & $6 \cdot 46 \pm 1 \cdot 14(100 \%)$ & $6 \cdot 62 \pm 1 \cdot 05(100 \%)$ & $0 \cdot 135$ \\
Enterococcus spp. & $6 \cdot 68 \pm 0 \cdot 75(100 \%)$ & $6 \cdot 58 \pm 0 \cdot 65(100 \%)$ & $0 \cdot 186$ \\
Streptococcus spp. & $8 \cdot 00 \pm 0 \cdot 20(25 \%)$ & $8 \cdot 00 \pm 0 \cdot 20 \quad(50 \%)$ & $0 \cdot 171$ \\
Stapylococcus spp. & $5 \cdot 14 \pm 0 \cdot 37(100 \%)$ & $4 \cdot 95 \pm 0 \cdot 45(100 \%)$ & $0 \cdot 18$ \\
Anaerobes & & & \\
Lactobacillus spp. & $8 \cdot 39 \pm 0 \cdot 79(100 \%)$ & $8 \cdot 40 \pm 0 \cdot 98(100 \%)$ & $0 \cdot 945$ \\
Bifidobacterium & $9 \cdot 28 \pm 0 \cdot 41(100 \%)$ & $9 \cdot 03 \pm 0 \cdot 08(100 \%)$ & $0 \cdot 128$ \\
spp. & & & \\
Clostridium spp. & $7 \cdot 41 \pm 0 \cdot 24(100 \%)$ & $7 \cdot 55 \pm 0 \cdot 55(100 \%)$ & $0 \cdot 181$ \\
Peptostreptococcus & $9 \cdot 11 \pm 0 \cdot 22(100 \%)$ & $9 \cdot 13 \pm 0 \cdot 23(100 \%)$ & $0 \cdot 598$ \\
spp. & & & \\
Veillonella spp. & $5 \cdot 60 \pm 0 \cdot 21(100 \%)$ & $5 \cdot 70 \pm 0 \cdot 20(100 \%)$ & $0 \cdot 24$ \\
Bacteroidaceae & $9 \cdot 60 \pm 0 \cdot 49(100 \%)$ & $9 \cdot 29 \pm 0 \cdot 33(100 \%)$ & $0 \cdot 104$ \\
\hline
\end{tabular}

${ }^{*}$ Number of organisms measured as (mean $\log _{10} \pm \mathrm{SD}$ ) per gram faeces. Detection incidence in all of macaques is shown in parentheses.

had almost recovered to the pretreatment level by day 15 . In this study, yeast, Pseudomonas aeruginosa and Clostridium difficile were not detected.

\section{Physical status of the macaques during the experiment}

The stool of an experimental macaque in the usual condition is solid and rather hard. All the macaques in the treatment group had soft stools during the experiment but no macaque had watery diarrhoea. Neither behavioural changes nor appetite loss was observed.

\section{DISCUSSION}

The composition of an individual's intestinal microflora basically remains constant, although it is known to fluctuate because of various environmental factors, which include not only antimicrobial treatment but also food, stress and, sometimes, weather changes (Simon \& Gorbach, 1984; Sullivan et al., 2001). However, appropriate identification of the microflora is methodologically not simple, as it requires meticulous anaerobic techniques (Simon \& Gorbach, 1984). Specifically, collected stool samples should be immediately put into an anaerobic transporter, transferred under anaerobic conditions and be analysed without delay.

Many studies have shown the influence of drugs, including antimicrobials, on the composition pattern of intestinal microflora (Brismar et al., 1991, 1993; Edlund et al., 1994, 2000a, b; Floor et al., 1994; Stark et al., 1996). Also, how eradication treatment for $H$. pylori infections affects intestinal microflora has been described (Adamsson et al., 1999; Bühling et al., 2001). However, because studies were per- formed on human subjects, methodological weaknesses could not be overcome, especially with respect to anaerobic techniques. In fact, reports on how antimicrobial treatment affects the profile of human anaerobic intestinal microflora do not necessarily reach agreement. For instance, amoxycillin is generally thought to have little effect on anaerobic flora (Brismar et al., 1993; Floor et al., 1994; Stark et al., 1996), while one report noted that only Lactobacillus and Bifidobacterium were suppressed by this antibiotic (Edlund et al., 1994). Further discordance is recognized regarding the effect of clarithromycin. Some reports cited that only a few anaerobic bacteria and not total anaerobic flora was suppressed during clarithromycin administration (Brismar et al., 1991; Edlund et al., 2000a), while another report showed that the total number of anaerobic bacteria decreased (Edlund et al., 2000b).

These discrepant observations in humans may partially come from methodological difficulties. With this background, we investigated changes in intestinal flora by antimicrobial treatment for $H$. pylori infections using Japanese macaques. Our study using this methodology has both merits and demerits.

One of the merits of this experimental animal is that its intestinal flora is known to be quite similar to that of human beings (Benno et al., 1987; Mitsuoka \& Kaneuchi, 1977). In addition, we could analyse stool samples taken directly from the rectum, which enables use of an anaerobic technique. Furthermore, strict control of diet and environmental conditions in these experimental animals could minimize environmental bias.

On the other hand, using experimental animals might be a limitation, as the delivery, distribution and metabolization of the antimicrobials may differ from that in humans, and the amounts of the drugs given in our experiment may represent an overdose for macaques (body weight about $9 \mathrm{~kg}$ ) since the amount was determined based on human use. Yet, higher doses of treatment drugs might be preferable since adverse effects of such treatment should be enhanced. In this experiment we used animals that were not infected with $H$. pylori. Regarding this concern, we previously reported that the intestinal microflora in $H$. pylori-infected macaques did not differ from those not infected (Fukuda et al., 1998).

Alterations in the composition of intestinal microflora were observed in this study during antimicrobial treatment; previous studies also made this observation. Adamsson et al. (1999) and Bühling et al. (2001) showed the change of human intestinal microflora following $H$. pylori treatment with omeprazole/metronidazole/amoxycillin and omeprazole/metronidazole/clarithromycin, respectively. Both demonstrated a marked decrease in anaerobic bacteria during treatment, but this decrease had almost reversed 35 days after the initiation of therapy. However, the eradication regimens used in both reports included metronidazole, which is not approved for H. pylori eradication therapy by the Japanese social security foundation. Metronidazole is known to possess potent suppressive effects, especially on 
anaerobic bacteria, and regimens that do not contain this antimicrobial may have a different effect on bacterial flora. Yet literature on regimens without metronidazole is not available. Therefore, we investigated the effect of the PPI/ amoxycillin/clarithromycin regimen, which is one of the most popular regimens in the world (Sharma et al., 1999). In our study, the total number of anaerobic bacteria did not change by day 8 , although specific species such as Lactobacillus and Bifidobacterium significantly decreased. These observations suggest that various combinations of antimicrobials yield different alterations in the composition of intestinal microflora.

Generally, diarrhoea and soft stools are considered to be results of disturbances of intestinal microflora, although the mechanism for diarrhoea is not fully understood (Bartlett, 2002). In clinical reports on diarrhoea and soft stools due to eradication treatment for $H$. pylori infection, the prevalence of these side effects differed according to the combination of antimicrobials. Lind et al. (1996) demonstrated in their MACH 1 (metronidazole, amoxicillin, clarithromycin, $H$. pylori, 1 week therapy) study that diarrhoea and soft stools appeared in $12 \cdot 1-17.9 \%$ of the patients treated with metronidazole-containing PPI-based regimens, whereas these side effects were seen in $26 \cdot 6-30 \cdot 0 \%$ of those on regimens without metronidazole. Misiewicz et al. (1997) reported that the prevalences of diarrhoea and soft stool in two different regimens using metronidazole and one regimen without metronidazole were $4 \cdot 2 \%, 9 \cdot 2 \%$ and $18 \cdot 2 \%$, respectively. These observations suggest that regimens without metronidazole may induce diarrhoea or soft stool more often than those with this drug. These findings, together with our results that anaerobic bacteria were not suppressed during treatment, suggest that whether anaerobic flora is suppressed may relate to presentation of diarrhoea or soft stools.

It should be noted that the alterations in bacterial microflora had almost reversed 7 days after completion of the treatment, suggesting that the clinically unfavourable effects such as diarrhoea or soft stools would stop in a short time. In fact, it is clinically well-known that the adverse effects of eradication treatment do not last for long periods (Armuzzi et al., 2001; Cremonini et al., 2002; Lind et al., 1996). Alterations in intestinal bacterial microflora in macaques by combination treatment for $H$. pylori infections supported this clinical experience.

\section{REFERENCES}

Adamsson, I., Nord, C. E., Lundquist, P., Sjöstedt, S. \& Edlund, C. (1999). Comparative effects of omeprazole, amoxycillin plus metronidazole versus omeprazole, clarithromycin plus metronidazole on the oral, gastric and intestinal microflora in Helicobacter pylori-infected patients. J Antimicrob Chemother 44, 629-640.

Armuzzi, A., Cremonini, F., Ojetti, V. \& 9 other authors (2001). Effect of Lactobacillus GG supplementation on antibiotic-associated gastrointestinal side effects during Helicobacter pylori eradication therapy: a pilot study. Digestion 63, 1-7.
Asaka, M., Satoh, K., Sugano, K. \& 7 other authors (2001). Guidelines in the management of Helicobacter pylori infection in Japan. Helicobacter 6, 177-186.

Bartlett, J. G. (2002). Clinical practice. Antibiotic-associated diarrhea. N Engl J Med 346, 334-339.

Benno, Y., Itoh, K., Miyao, Y. \& Mitsuoka, T. (1987). Comparison of fecal microflora between wild Japanese monkeys in a snowy area and laboratory-reared Japanese monkeys. Jpn J Vet Sci 49, 1059-1064 (in Japanese).

Brismar, B., Edlund, C. \& Nord, C. E. (1991). Comparative effects of clarithromycin and erythromycin on the normal intestinal microflora. Scand J Infect Dis 23, 635-642.

Brismar, B., Edlund, C. \& Nord, C. E. (1993). Impact of cefpodoxime proxetil and amoxicillin on the normal oral and intestinal microflora. Eur J Clin Microbiol Infect Dis 12, 714-719.

Bühling, A., Radun, D., Müller, W. A. \& Malfertheiner, P. (2001). Influence of anti-Helicobacter triple-therapy with metronidazole, omeprazole and clarithromycin on intestinal microflora. Aliment Pharmacol Ther 15, 1445-1452.

Cremonini, F., Di Caro, S., Covino, M. \& 7 other authors (2002). Effect of different probiotic preparations on anti-Helicobacter pylori therapyrelated side effects: a parallel group, triple blind, placebo-controlled study. Am J Gastroenterol 97, 2744-2749.

Edlund, C., Stark, C. \& Nord, C. E. (1994). The relationship between an increase in beta-lactamase activity after oral administration of three new cephalosporins and protection against intestinal ecological disturbances. J Antimicrob Chemother 34, 127-138.

Edlund, C., Alván, G., Barkholt, L., Vacheron, F. \& Nord, C. E. (2000a). Pharmacokinetics and comparative effects of telithromycin (HMR 3647) and clarithromycin on the oropharyngeal and intestinal microflora. J Antimicrob Chemother 46, 741-749.

Edlund, C., Beyer, G., Hiemer-Bau, M., Ziege, S., Lode, H. \& Nord, C. E. (2000b). Comparative effects of moxifloxacin and clarithromycin on the normal intestinal microflora. Scand J Infect Dis 32, 81-85.

Floor, M., van Akkeren, F., Rozenberg-Arska, M., Visser, M., Kolsters, A., Beumer, H. \& Verhoef, J. (1994). Effect of loracarbef and amoxicillin on the oropharyngeal and intestinal microflora of patients with bronchitis. Scand J Infect Dis 26, 191-197.

Fukuda, Y., Tamura, K., Yamamoto, I., Kawaura, A., Tonokatsu, W., Tsuyuguchi, T., Ohno, T., Satomi, M. \& Shimoyama, T. (1992). Inoculation of rhesus monkeys with human Helicobacter pylori: a long-term investigation on gastric mucosa by endoscopy. Dig Endosc 4, 19-30.

Fukuda, Y., Shintani, S., Okui, M., Yamamoto, I., Tamura, K. \& Shimoyama, T. (1998). Intestinal microflora after eradication treatment for Helicobacter pylori infection in Japanese monkeys. Syouka to Kyusyu 21, 65-68 (in Japanese).

Högenauer, C., Hammer, H. F., Krejs, G. J. \& Reisinger, E. C. (1998). Mechanisms and management of antibiotic-associated diarrhea. Clin Infect Dis 27, 702-710.

Lind, T., Veldhuyzen van Zanten, S., Unge, P. \& 8 other authors (1996). Eradication of Helicobacter pylori using one-week triple therapies combining omeprazole with two antimicrobials: the MACH 1 study. Helicobacter 1, 138-144.

Misiewicz, J. J., Harris, A. W., Bardhan, K. D. \& 7 other authors (1997). One week triple therapy for Helicobacter pylori: a multicentre comparative study. Gut 41, 735-739.

Mitsuoka, T. (1980). The world of intestinal bacteria. In A Color Atlas of Anaerobic Bacteria. Tokyo: Sohbunsya (in Japanese).

Mitsuoka, T. \& Kaneuchi, C. (1977). Ecology of the bifidobacteria. Am J Clin Nutr 30, 1799-1810. 
Murray, P. R., Baron, E. J., Pfaller, M. A., Tenover, F. C. \& Yolken, R. H. (1999). Manual of Clinical Microbiology, 7th edn. Washington, DC; American Society for Microbiology.

Sharma, V. K., Vasudeva, R. \& Howden, C. W. (1999). A survey of gastroenterologists' perceptions and practices related to Helicobacter pylori infection. Am J Gastroenterol 94, 3170-3174.

Simon, G. L. \& Gorbach, S. L. (1984). Intestinal flora in health and disease. Gastroenterology 86, 174-193.

Stark, C. A., Adamsson, I., Edlund, C., Sjösted, S., Seensalu, R., Wikström, B. \& Nord, C. E. (1996). Effects of omeprazole and amoxycillin on the human oral and gastrointestinal microflora in patients with Helicobacter pylori infection. J Antimicrob Chemother 38 927-939.
Sullivan, A., Edlund, C. \& Nord, C. E. (2001). Effect of antimicrobial agents on the ecological balance of human microflora. Lancet Infect Dis $1,101-114$

Uemura, N., Okamoto, S., Yamamoto, S., Matsumura, N., Yamaguchi, S., Yamakido, M., Taniyama, K., Sasaki, N. \& Schlemper, R. J. (2001). Helicobacter pylori infection and the development of gastric cancer. $N$ Engl J Med 345, 784-789.

Van der Hulst, R. W., Rauws, E. A., Köycü, B., Keller, J. J., Bruno, M. J., Tussen, J. G. \& Tytgat, G. N. (1997). Prevention of ulcer recurrence after eradication of Helicobacter pylori: a prospective long-term follow-up study. Gastroenterology 113, 1082-1086.

Wotherspoon, A. C., Doglioni, C., Diss, T. C., Pan, L., Moschini, A., der Boni, M. \& Isaacson, P. G. (1993). Regression of primary low-grade Bcell gastric lymphoma of mucosa-associated lymphoid tissue type after eradication of Helicobacter pylori. Lancet 342, 575-577. 